\title{
Sobre las restricciones distribucionales de casi $^{*}$
}

\author{
Raquel González Rodríguez - Universidad Complutense de Madrid \\ raquelgonzalezr@ucm.es
}

Rebut / Received: 6/09/19

Acceptat / Accepted: 6/12/19

Resum. Sobre les restriccions distribucionals de casi. Aquest treball estudia les restriccions distribucionals que presenta l'adverbi d'aproximació casi en contextos negatius. Des d'un punt de vista descriptiu, es mostra que casi és incompatible amb la negació quan modifica a una expressió numeral i que presenta la situació contrària si incideix sobre substantius comptables en plural o substantius incomptables. Quan precedeix a aquest tipus de substantius, casi requereix la presència de la negació. Des d'un punt de vista teòric, s'ofereix una explicació d'aquestes restriccions basada en les propietats semàntiques de casi i en les dels contextos amb els que és incompatible.

Paraules clau: negació, termes de polaritat, contextos decreixents, adverbis d'aproximació.

Abstract. On the distributional restrictions of casi $^{\text {' }}$ almost'. This paper studies the distributional restrictions that the approximative adverb casi ('almost') displays. From a descriptive point of view, it is shown that casi is incompatible with negation when this adverb modifies a numeral word and that it requires the presence of negation if it precedes countable nouns in plural or uncountable nouns. From a theoretical point of view, this paper offers an explanation of these restrictions based on the semantic properties of cassi and the environments with which this item is incompatible.

Keywords: negation, polarity items, download contexts, approximative adverbs.

\footnotetext{
* Agradezco enormemente a los dos revisores anónimos sus atinados comentarios y sugerencias, que han contribuido a mejorar la versión final de este trabajo. Los errores que pudieran persistir son solo a mí atribuibles.
} 


\section{Introducción}

Casi constituye un adverbio de aproximación, esto es, un adverbio que modifica la extensión del término sobre el que incide señalando una propiedad próxima a la denotada por dicho término (Moreno Cabrera, 1984; RAE, 2009). En (1a), casi no denota que alguien posea la propiedad de ser alto en un determinado grado, sino que tiene una propiedad cercana a esa. De esta descripción se sigue que casi no pueda adscribirse a la clase de los adverbios de grado, entre los que se encuentran los cuantificadores de (1b), que sí adscriben la propiedad de ser alto al individuo al que refiera el sujeto de predicación.
(1) a. Es casi alto.
b. Es $\{$ bastante / muy\} alto.

La diferencia entre casi y los adverbios de grado se pone de manifiesto en la posibilidad de modificar a adjetivos no graduables. Casi, a diferencia de los adverbios de grado, es compatible con ese tipo de adjetivos (2), lo que indica que no expresa el grado en que se posee una propiedad.
(2) a. Es casi universitario.
b. ${ }^{*}$ Es $\{$ bastante / muy $\}$ universitario.

Como señala Moreno Cabrera (1984), casi no solo puede incidir sobre adjetivos, sino que puede hacerlo sobre un buen número de categorías gramaticales. Como se muestra en (3a), entre los elementos a los que puede modificar casi se encuentran los nombres escuetos, los sintagmas nominales introducidos por un indefinido, los sintagmas preposicionales, los adverbios o los sintagmas verbales. Este adverbio no puede, en cambio, incidir sobre la mayoría de los sintagmas nominales encabezados por un artículo, sobre los nombres propios o sobre los pronombres (3b). A partir de contrastes como el de (3a) y (3b), Moreno Cabrera (1984) propone que casi modifica a elementos que funcionan como predicados, pero no a constituyentes referenciales.
(3) a. casi \{niño/ un niño/ de Madrid/ siempre/ lo hizo\}
b. * casi $\{$ el niño/ Juan/ yo $\}$

(Moreno Cabrera, 1984, p. 243-244)

Este trabajo se va a centrar en los casos en que casi modifica a un numeral, a un sustantivo discontinuo o a un sustantivo contable en plural:

(4) casi $\{$ veinte amigos/ agua/ votos\} 
Estas construcciones resultan de interés porque presentan una distribución opuesta. Obsérvense los siguientes ejemplos:

(5) a. $\left({ }^{*} \mathrm{No}\right)$ tiene casi veinte amigos. ${ }^{1}$

b. ${ }^{*}(\mathrm{No})$ queda casi agua.

c. ${ }^{*}(\mathrm{No})$ ha conseguido casi votos.

En (5a), donde casi modifica a una expresión numeral, el constituyente casi veinte amigos requiere aparecer en una oración positiva, como muestra el que la presencia de la negación dé lugar a una oración agramatical (González Rodríguez, 2008; Spector, 2014). Los ejemplos de (5b) y (5c), en los que el adverbio incide sobre un sustantivo incontable y sobre un sustantivo contable en plural, respectivamente, presentan la situación contraria: si la negación no está presente, las oraciones están mal formadas. La única diferencia entre (5a), por un lado, y (5b)-(5c), por otro, está en el tipo de elemento al que modifica casi. ${ }^{2}$

Las restricciones ilustradas en (5) han sido ampliamente estudiadas por autores como Bosque (1980), Sánchez López (1999), Szabolcsi (2004) y Giannakidou (2011), entre otros. El mismo comportamiento que casi veinte amigos lo presentan los elementos que aparecen en cursiva en (6). A los elementos que no pueden estar bajo el alcance de la negación se los denomina términos de polaridad positiva (TPPs). Se comportan como casi agua y casi votos los que se destacan en (7). Todos ellos se caracterizan por necesitar estar en el ámbito de la negación, por lo que reciben el nombre de términos de polaridad negativa (TPNs).

(6) ¡Qué rápido (*no) habla!

b. $\left({ }^{*} \mathrm{No}\right)$ vendió como poco un millón de discos.

(7) a. *(No) encontró errata alguna.

b. *(No) abrazó a nadie.

La necesidad de aparecer en entornos positivos o negativos no es, por tanto, una propiedad inusual. Lo que sí resulta sorprendente es que un mismo elemento se comporte como un TPP unas veces y como un TPN otras (5). Además, cuando casi modifica a un sintagma verbal, no presenta ninguna restricción distribucional vinculada con la polaridad oracional. En ese contexto, casi puede aparecer tanto en una oración afirmativa como

1. La negación es posible si interpretamos que se está refutando una construcción emitida previamente en el discurso o que se presupone. Esta lectura debe ser descartada a lo largo de todo el trabajo. Asimismo, si casi escapa al alcance de la negación, deja de ser incompatible con ella.

2. Como indican Fábregas (2015) y González Rodríguez (2008), apenas presenta el mismo comportamiento.

3. Cabe señalar que no siempre que casi va seguido de un sustantivo contable en plural o un sustantivo incontable tenemos un TPN. Se estudiará con más detenimiento esta cuestión en el apartado 3.1. 
en una negativa, aunque en este último caso siempre tiene alcance amplio con respecto a la negación. La posibilidad de que casi incida sobre sintagmas verbales afirmativos y negativos se ilustra en (8). Cuando no está presente la negación se sigue que el evento denotado por el sintagma verbal no ha tenido lugar mientras que, cuando aparece no, se sigue lo contrario, esto es, que la persona en cuestión ha aprobado el examen.

(8) Casi (no) aprueba el examen.

El propósito de este trabajo es precisamente explicar tanto por qué casi no puede aparecer en el ámbito de la negación cuando incide sobre una expresión numeral como por qué presenta la distribución opuesta si precede a un nombre no contable o a uno contable en plural. Propondremos que la limitada distribución de casi veinte amigos, casi agua y casi votos responde a que las propiedades semánticas de esos sintagmas son incompatibles con las de los contextos en que no pueden aparecer, en línea con Kadmon y Landman (1993), Lahiri (1998) y Chierchia (2006).

Este trabajo se divide como sigue. En el apartado 2 se estudia en qué clases de entornos negativos son rechazados los constituyentes <casi + expresión numeral> y en cuáles son legitimados los formados por casi seguido de un sustantivo contable en plural o de un sustantivo discontinuo. En el apartado 3 se explican las restricciones distribucionales descritas en el apartado anterior. En el apartado 4 se presentan las conclusiones del trabajo.

\section{Casi en los contextos negativos}

Los contextos negativos son aquellos que legitiman TPNs y rechazan la presencia de TPPs dentro de su alcance. Esto es lo que sucede, como ya hemos visto, con la partícula no. Sin embargo, este no es el único elemento que da lugar a un contexto negativo. Existen otros muchos que presentan un comportamiento similar. Autores como Dowty (1994), Hoeksema (1983), Ladusaw (1979), van der Wouden (1997) y Zwarts (1998) han propuesto que existen tres clases de entornos negativos: los contextos decrecientes, los antiaditivos y los antimórficos. Estos mantienen entre sí la siguiente relación de inclusión: los antimórficos y los antiaditivos son un subconjunto de los decrecientes y los primeros son, a su vez, un subconjunto de los segundos.

Los entornos decrecientes dan lugar a inferencias de conjuntos a subconjuntos. Se comportan así, por ejemplo, poco(s) y raramente. Si tomamos dos conjuntos, el de las verduras y el de las espinacas, que constituye un subconjunto del primero, poco desencadena inferencias del conjunto al subconjunto, puesto que una oración como Pocos niños comen verduras implica que Pocos niños comen espinacas.

Entre las construcciones antiaditivas están la preposición sin, las palabras negativas (ninguno, nadie, etc.) y los complementos proposicionales de los predicados negativos (negar, dudar, etc.). Estas construcciones se caracterizan por que la construcción que 
resulta si la función incide sobre la disyunción de dos elementos es equivalente a la que surge de la conjunción de dos construcciones, aquellas en que la función modifica a cada uno de los elementos mencionados por separado. En otras palabras, sin es una función antiaditiva porque (9a), donde la preposición incide sobre la disyunción de dos constituyentes, despedirse y decir dónde iba, es equivalente a (9b), donde tenemos la conjunción de las construcciones en las que sin incide sobre cada uno de esos sintagmas:

(9) a. Juan se marchó sin despedirse o decir dónde iba.

b. Juan se marchó sin despedirse y sin decir dónde iba.

No es el único elemento que posee la propiedad de ser antimórfico. Su adscripción a ese tipo de funciones obedece a que satisface dos requisitos. En primer lugar, cumple la condición que caracteriza a las funciones antiaditivas, puesto que (10a) es equivalente a (10b); en segundo lugar, la negación de la conjunción de dos elementos es equivalente a la disyunción de la negación de cada uno de esos elementos (11), es decir, la oración de (11a) equivale a la de (11b):

(10) a. Juan no se despidió o dijo dónde iba.

b. Juan no se despidió y no dijo dónde iba.

(11) a. Juan no se despidió y dijo dónde iba.

b. Juan no se despidió o no dijo dónde iba.

No todos los términos de polaridad son sensibles a los mismos entornos negativos. Como se muestra en (13), tanto el cuantificador exclamativo qué simpático como el adverbio extremadamente son incompatibles con la negación y, por tanto, TPPs. Ahora bien, eso no significa que sean rechazados en los mismos contextos negativos. El adverbio raramente da lugar a un contexto decreciente al que es sensible el cuantificador exclamativo (véase (12a)). A diferencia de lo que sucede con qué simpático, el TPP extremadamente no es incompatible con el operador decreciente raramente, lo que muestra que no todos los TPPs son rechazados en los mismos contextos. Qué simpático es sensible a los entornos decrecientes mientras que extremadamente lo es a los antimórficos.

(12) a. ¡Qué simpático (*raramente) está Pedro!

b. Sus alumnos raramente son extremadamente irresponsables.

(13) a. ¡Qué simpático (*no) está Pedro!

b. Sus alumnos ( ${ }^{*}$ no) son extremadamente irresponsables. ${ }^{4}$

4. Nótese que esta oración es gramatical si añadimos una continuación como son solo un poco irresponsables (Sus alumnos no son extremadamente irresponsables, son solo un poco irresponsables). La razón es que en este caso 
En la introducción señalamos que casi se comporta en unos casos como un TPN y en otros como un TPP, pero únicamente tuvimos en cuenta su (in)compatibilidad con no. A continuación describimos qué sucede cuando casi aparece en otros contextos negativos. Comencemos por determinar qué sucede con el TPN <casi + expresión numeral $>$. En lo que respecta a los contextos antimórficos, ya se ha mostrado que ese constituyente es rechazado en esos entornos, puesto que no puede aparecer en el ámbito de la negación.

(14) *No fuma casi dos paquetes de tabaco al día.

Pasemos ahora a observar qué sucede en los contextos antiaditivos y los decrecientes. Cuando casi modifica a una expresión numeral, es incompatible con ambos, como se muestra en (15) y (16), respectivamente.

(15) a. ${ }^{? / *}$ Ninguno de esos libros tiene casi trescientas páginas.

b. ?"** Se arregló sin tardar casi dos horas.

(16) * (Pocos niños/ raramente $\}$ leen casi dos libros al mes.

En lo que respecta a casi con sustantivos contables en plural y con nombres discontinuos, ya señalamos que estos sintagmas pueden estar legitimados por no, esto es, por los entornos antimórficos.

(17) a. No ha obtenido casi apoyos.

b. No toma casi azúcar.

La situación es distinta en los entornos antiaditivos. Ni las palabras negativas (18) ni un verbo como dudar (19) legitiman el TPN casi.

(18) a. *Ninguno ha leído casi libros.

b. *Ninguno toma casi azúcar.

(19) a. *Dudo que haya leído casi libros.

b. *Dudo que tome casi azúcar.

En lo que respecta a sin, resulta difícil comprobar si legitima el TPN casi. La razón es que construcciones como las de (20), donde casi está bajo el alcance de sin, resultan

estamos ante una negación externa o metalingüística, es decir, se está refutando la correspondiente oración afirmativa (Sus alumnos son extremadamente irresponsables) y el foco contrastivo recae sobre el cuantificador. Como señalamos en la nota 1 , en estos casos, los TPPs no son incompatibles con la negación. 
muy forzadas y en el orden inverso (véase (21)), casi modifica al SP, por lo que escapa al alcance de $\sin$.

(20) a. "Montó el mueble sin casi herramientas.

b. "Toma el café sin casi azúcar.

(21) a. Montó el mueble casi sin herramientas.

b. Toma el café casi sin azúcar.

Los entornos decrecientes tampoco legitiman la presencia del TPN casi.

(22) a. *Pocos niños leen casi dos libros al mes.

b. *Mi madre raramente toma casi azúcar.

Los datos que hemos introducido en esta sección muestran que el TPP casi es incompatible con los contextos decrecientes, mientras que, cuando casi se comporta como un TPN, solo queda legitimado por no (contextos antimórficos).

\section{Análisis}

En este apartado ofrecemos un análisis semántico de la limitada distribución de casi tanto como TPN como como TPP. En el apartado 3.1 se proporciona un análisis semántico de casi. En el apartado 3.2 se da cuenta de la limitada distribución de <casi + expresión numeral>, esto es, del TPP casi. En el apartado 3.3 se explica por qué <casi + nombres contables en plural / nombres incontables> requiere estar en el ámbito de la negación.

\subsection{La semántica de casi}

Siguiendo a Penka (2006), propondremos un análisis de casi basado en las alternativas escalares que surgen con respecto al elemento sobre el que incide. Este modificador desencadena la interpretación escalar de dicho elemento. De este modo, el constituyente sobre el que incide debe poder interpretarse con respecto a un modelo escalar, es decir, como un valor que forma parte de una determinada escala (Nouwen, 2000). Cuando casi modifica una expresión numeral, se trata de una escala numeral, como en (23), donde se indica que el número de puntos obtenidos fue menor de cien pero cercano a ese valor. ${ }^{5}$

5. Este es el uso que Pons y Schwenter (2011) denominan canónico. Como notan estos autores, en ciertos contextos casi puede también dar lugar a una lectura descendente de la escala en que se señala un punto superior al que refiere el elemento modificado por el aproximativo. Aunque no nos centraremos en este uso, consideramos que nuestro análisis puede aplicarse igualmente a esos casos (véase el apartado 3.3, donde se 
(23) Obtuvo casi cien puntos.

El mismo análisis puede aplicarse a los casos en que casi modifica otro tipo de elementos. Como señala la RAE (2009), en (24) puede concebirse igualmente una escala. La diferencia es que se trata de una jerarquía de propiedades.

(24) Es casi ilegal.

En el caso de los nombres contables en plural y los discontinuos en singular, tenemos una jerarquía de propiedades o de cantidad en función del contexto. Obsérvense los siguientes ejemplos:

(25) a. Esa sustancia es casi agua.

b. Esas canciones son casi poemas.

(26) a. No tiene casi agua.

b. No ha escrito casi poemas.

En (25), casi se interpreta con respecto a una jerarquía de propiedades. Así, (26a) es adecuada, por ejemplo, en un contexto en que se está realizando un experimento para obtener agua a partir de ciertos elementos. Esa oración podría expresarse si el resultado de una de las combinaciones da un líquido que no puede denominarse agua pero que es muy cercano a esta. La misma lectura surge en (25b), donde se expresa que las canciones a las que se alude no son poemas pero sí algo muy cercano a dicha composición. La interpretación de estos ejemplos es, por tanto, paralela a la que tenemos en (24). En (26), en cambio, se alude a una jerarquía de cantidad, de forma que estas construcciones son más cercanas a la de (23) que a la de (24). En estos casos no se alude a algo que no sea agua o poemas pero que se parezca a estas entidades. A lo que se alude es a una determinada cantidad de agua o poemas. En concreto, a que la cantidad no es cero pero sí un valor cercano a esta cifra.

Además del tipo de escala a la que se asocian, los ejemplos de (25) y (26) se diferencian en otro aspecto: solo se comportan como TPNs los sintagmas encabezados por casi de (26). Los propios ejemplos de (25), en los que no está presente la negación, ponen de manifiesto que en este caso no estamos ante un TPN, aunque casi modifica a un nombre contable en plural (25b) y a uno discontinuo (25a). Este contraste indica que la generalización que habíamos establecido hasta ahora era demasiado amplia. Para que tengamos un TPN, casi no solo debe modificar a un sustantivo contable en plural o a uno discontinuo, sino que la escala con la que se asocia casi debe ser una escala de cantidad.

tratan casos similares). Pons y Schwenter (2011) también aluden a una interpretación en que casi modifica la fuerza ilocutiva y que es analizada en Pardo Llibrer (2018). 
Volvamos ahora a la interpretación de casi. Consideramos que la interpretación de casi está estrechamente vinculada con el conjunto de alternativas que surge de la interpretación escalar del elemento modificado. Siguiendo a Penka (2006), defendemos que la función de casi es expresar si ciertas alternativas del modelo escalar relevante cumplen o no las condiciones de verdad impuestas por la proposición. Como se recoge en (27), casi indica que la alternativa introducida por el elemento modificado no satisface el contenido proposicional de la oración, pero que alguna inferior y cercana a él sí (en (27), el símbolo $<$ significa 'inferior y cercano'). ${ }^{6}$

$$
[[\operatorname{casi} \mathrm{x}(\mathrm{P})]]=1 \operatorname{ssi} \neg \mathrm{P}(\mathrm{x}) \wedge \exists \mathrm{y}[\mathrm{y}<\approx \mathrm{x} \wedge \mathrm{P}(\mathrm{y})]^{7}
$$

Nótese que este análisis recoge las condiciones de verdad de los ejemplos ofrecidos antes. La proposición correspondiente a una oración como la de (23), por ejemplo, será verdadera si el número de puntos obtenidos no es cien sino una cantidad cercana a esta cifra por la parte inferior de la escala. Lo mismo se aplica a un ejemplo como el de (24). La única diferencia es que no se trata de una escala numeral sino de una de propiedades.

\subsection{Casi como TPP}

Una vez hemos expuesto el análisis semántico de casi, explicaremos por qué este modificador se comporta como un término de polaridad. En este apartado nos centraremos en su comportamiento como TPP (<casi + expresión numeral $>$ ). Dejamos para el siguiente apartado, el 3.3, los casos en que casi funciona como un TPN.

Como mostramos en el apartado 2, el constituyente <casi + expresión numeral> es incompatible con los contextos decrecientes. Estos entornos establecen dos clases de inferencias en las escalas numerales: por una parte, los valores jerárquicamente superiores implican lógicamente los inferiores; por otra, a partir de los inferiores surge una implicatura conversacional según la cual se niegan los superiores. De este modo, una oración como Pesa setenta kilos desencadena dos inferencias. Respecto a los valores inferiores de la escala, se implica que ellos también satisfacen el contenido proposicional de la oración (28). Esta inferencia es una implicación lógica, ya que no puede ser cancelada (29): ${ }^{8}$

6. Consúltese Penka (2006) para una revisión crítica de los análisis de almost propuestos por Morzycki (2001) y Sadock (1981).

7. Por razones de espacio no entraremos a discutir si alguna de las partes de este significado forma parte de la aserción, constituye parte de una presuposición... (Atlas, 1996; Ziegeler, 2000, 2010; Horn, 2002; Amaral, 2007).

8. Para que esta implicación lógica tenga lugar es necesario interpretar que se está expresando el valor escalar alcanzado por la entidad denotada por el sujeto de predicación, y no el que posee exactamente. 
(28) Pesa setenta kilos $\rightarrow$ pesa sesenta kilos.

(29) Pesa setenta kilos, \#pero no pesa sesenta kilos.

Con respecto a los valores superiores se infiere lo contrario, es decir, que no satisfacen el contenido proposicional de la oración. En otras palabras, al afirmar que un individuo alcanza los setenta kilos se implica conversacionalmente que no pesa ochenta kilos (30a). Frente a lo que sucedía con la inferencia anterior, esta puede ser cancelada, lo que muestra que es una implicatura conversacional. La posibilidad de cancelar esta inferencia se pone de manifiesto en (30b), donde se afirma que un individuo alcanza los setenta kilos e incluso los ochenta. Imaginemos un contexto en que se está eligiendo atletas para una determinada prueba y que el principal requisito es que pesen como mínimo setenta kilos. En ese contexto, la persona encargada de pesar a los deportistas e indicar si pasan o no el criterio podría expresar (30b). De este modo, si bien de una construcción como Pesa setenta kilos se infiere que no se alcanzan los ochenta, esta no es una implicación lógica, dado que, como acabamos de explicar, se puede cancelar.

(30) a. Pesa setenta kilos $\rightarrow$ no pesa ochenta kilos.

b. Pesa setenta kilos; de hecho, pesa ochenta kilos.

Como hemos propuesto, casi expresa que la alternativa a la que refiere el elemento al que modifica da lugar a una proposición falsa, mientras que la proposición es cierta si tomamos en consideración el valor correspondiente a alguna alternativa cercana e inferior. El negar una alternativa y afirmar otra inferior a ella no plantea ningún problema en los contextos crecientes, dado que la afirmación de una alternativa no conlleva la de valores superiores a ella. Sin embargo, si casi aparece en un contexto decreciente, no podrá realizar su función, esto es, no podrá negar una alternativa y afirmar una inferior a ella al mismo tiempo. ${ }^{9}$ La razón reside en que los entornos decrecientes se caracterizan por invertir las inferencias escalares; el afirmar que no se ha alcanzado un determinado grado en la escala implica que no se han alcanzado los superiores. De este modo, los valores jerárquicamente superiores a otro son incluidos mediante una implicación lógica en estos contextos. Considérese, por ejemplo, la oración de (31), que contiene el cuantificador decreciente raramente. Frente a lo que sucedía con la oración sin dicho operador (28), (31) no implica lógicamente que el individuo en cuestión raramente alcance los sesenta kilos, sino que raramente alcanza los ochenta kilos (32):

(31) Raramente pesa setenta kilos.

9. El hecho de que la alternativa que se afirma deba también ser cercana a ella no conlleva ningún problema. 
(32) Raramente pesa setenta kilos $\rightarrow$ Raramente pesa ochenta kilos.

Pues bien, si introducimos casi en este contexto (33), estaríamos negando la alternativa escalar introducida por la expresión numeral a la que modifica casi y afirmando una inferior. Esto no es posible porque, como acabamos de mostrar, en los contextos decrecientes, la afirmación de una alternativa implica lógicamente la afirmación de las que son jerárquicamente superiores. Veamos cómo puede explicarse la agramaticalidad de (33) a partir de esto. Acabamos de señalar que si en pocas ocasiones una persona alcanza los setenta kilos de peso, en pocas ocasiones alcanza los ochenta. El problema en una oración como la de (33) es que esa implicación lógica es incompatible con la denotación de casi. La presencia de este modificador obliga a negar las alternativas que son jerárquicamente superiores a la denotada por el elemento al que modifican, lo que significa negar que raramente pesa ochenta kilos. La negación de esta alternativa no es posible en un contexto decreciente porque, como hemos dicho, está implicada lógicamente. Las alternativas que niega casi son las mismas que se implican lógicamente, lo que provoca la mala formación de la oración.

(33) *Raramente pesa casi setenta kilos.

La limitada distribución de casi como TPP obedece, por tanto, a que su semántica, basada en la inclusión/exclusión de ciertas alternativas de un modelo escalar, entra en conflicto con las inferencias desencadenadas por los contextos decrecientes.

\subsection{Casi como TPN}

En esta sección explicaremos las restricciones distribucionales que presenta casi cuando modifica a nombres contables en plural o a nombres discontinuos. Recuérdese que estos constituyentes necesitan estar en el ámbito de la negación cuando la escala con la que se asocia la interpretación del sintagma es una escala de cantidad (34).

(34) a. $\left({ }^{*} \mathrm{No}\right)$ tiene casi agua.

b. $\left({ }^{*} \mathrm{No}\right)$ ha escrito casi poemas.

Nuestra propuesta es que la agramaticalidad de (34) cuando no está presente la negación obedece a que, en ese caso, la denotación de agua y poemas es incompatible con la de casi si interpretamos la secuencia con respecto a una escala numeral. Con casi se alude a un valor de la escala inferior, pero cercano, al que refiere el elemento sobre el que incide el adverbio. Sin embargo, tanto agua como poemas poseen una denotación no delimitada, de forma que no aluden a ningún valor en una escala de cantidad o, en otras palabras, no indican una cantidad determinada de agua o de poemas (Bosque y 
Gutiérrez-Rexach, 2009) ${ }^{10}$. Como consecuencia, casi no puede incidir sobre ellos. Si no se alude a un determinado valor de la escala, casi no puede realizar su función, puesto que necesita un punto de la escala en relación con el que expresar cercanía por debajo.

La pregunta que surge ahora es por qué, si se introduce no, esas oraciones pasan a ser gramaticales. Obsérvense las oraciones siguientes:
(35) a. No tiene agua.
b. No ha escrito poemas.

En estos ejemplos sí se alude a una cantidad determinada de agua y de poemas, la correspondiente al punto más bajo de la escala, que no es otra que la ausencia de agua y poemas. El que se aluda a la ausencia de esas materias está determinado por la negación, que refuta que tenga agua y que haya escrito poemas. Como consecuencia, no legitima la presencia de casi, que sí puede señalar a un valor cercano al que refiere en ese contexto el elemento al que modifica. Por tanto, el hecho de que la negación legitime la presencia de esos constituyentes no está relacionado con que invierta las inferencias escalares. De hecho, el resto de elementos que crean entornos negativos no legitiman el TPN casi, como se mostró en el apartado 2.

Como se ha indicado, la legitimación de los TPNs que estamos analizando no está asociada con la inversión de las inferencias escalares. Ahora bien, esta inversión, a la que da lugar la negación, sí permite explicar otra propiedad de las construcciones de (34). Estos ejemplos parecen presentar una diferencia con respecto a otros en que aparece el adverbio de aproximación: el valor al que se alude en (34) es superior al asociado con (35), no inferior. En (34) se alude a cierta cantidad de agua y de poemas, mientras que en (35) no habría ni agua ni poemas. Esto parece contradictorio con la semántica de casi, ya que este modificador señala un punto de la escala inferior, aunque cercano, al introducido por el elemento al que modifica. A este respecto nos gustaría sugerir que los ejemplos de (34) no constituyen contraejemplos reales a la interpretación de casi propuesta. Si tenemos en cuenta que la negación invierte la dirección de las escalas, podría considerarse que (34) se ajusta al patrón general. Puesto que en los contextos decrecientes las implicaciones lógicas se producen hacia la parte alta de la escala, en ese tipo de entornos no tener nada de agua implica no tener ningún valor superior al mínimo. Por lo tanto, los valores jerárquicamente inferiores son informativamente más fuertes que los superiores. Adoptando esta perspectiva, en (34) casi aludiría a un valor jerárquicamente inferior al denotado en su ausencia.

10. Queda fuera de los límites de este trabajo un estudio detallado de la denotación de los sustantivos continuos en plural y de los discontinuos. Consúltese a este respecto el volumen editado por Bosque (1996). 


\section{Conclusiones}

En este trabajo se ha estudiado la distribución de casi en los contextos negativos. Se ha mostrado que rechaza la negación y los contextos decrecientes cuando incide sobre una expresión numeral, de forma que en estos casos se comporta como un TPP. Si precede a un sustantivo contable en plural o a un sustantivo incontable, necesita estar en el alcance de la negación, por lo que funciona como un TPN.

Una vez descritas las restricciones distribucionales de casi, se ha ofrecido una explicación de ellas. Se ha propuesto que estas obedecen a que las propiedades semánticas de <casi + expresión numeral> y <casi + sustantivo contable en plural / sustantivo incontable> son incompatibles con las de los contextos en que esos constituyentes son rechazados. En el caso de <casi + expresión numeral $>$, se ha defendido que la interpretación de casi es incompatible con el tipo de inferencias que se realizan en los contextos decrecientes. Ese modificador expresa que la alternativa introducida por el elemento modificado no satisface el contenido proposicional de la oración mientras que alguna alternativa inferior y cercana a esa sí. Se niega, por tanto, un valor de la escala numeral y se afirma uno inferior y cercano a él. Esto no es posible en los contextos decrecientes porque en ellos la afirmación de un valor implica lógicamente la afirmación de los valores superiores. En lo que respecta a <casi + sustantivo contable en plural / sustantivo incontable>, se ha argumentado que ese constituyente no puede aparecer en los contextos afirmativos porque esos sustantivos no aluden a ningún valor concreto de una escala de cantidad. Si no se hace referencia a un valor en una escala, casi no puede realizar su función, que es precisamente aludir a un valor inferior pero cercano al denotado por el elemento al que modifica. Cuando ese constituyente está bajo el alcance de la negación, se hace referencia a un determinado valor, el cero, y, por tanto, casi puede aparecer.

\section{Referencias}

Amaral, P. M. (2007). The meaning of approximative adverbs: Evidence from European Portuguese. (Tesis doctoral). The Ohio State University, Columbus.

Atlas, J. (1996). Only noun phrases, pseudo-negative generalized quantifiers, negative polarity items, and monotonicity. Journal of Semantics, 13, 265-332.

Bosque, I. (1980). Sobre la negación. Madrid: Cátedra.

Bosque, I. (Ed.) (1996).El sustantivo sin determinación. Madrid: Visor.

Bosque, I., y Gutiérrez-Rexach, J. (2009). Fundamentos de sintaxis formal. Madrid: Akal.

Chierchia, G. (2006). Broaden your views: Implicatures of domain widening and the "Logicality" of language. Linguistic Inquiry, 37, 535-590.

Dowty, D. (1994). The role of negative polarity and concord marking in natural language reasoning. En M. Harvey y L. Santelmann (Eds.), Proceedings of semantics and linguistic theory $I V$ (pp. 114-144). Itaca: Cornell University. 
Fábregas, A. (2015). Ni positivo ni negativo: una nota sobre apenas. Ogigia: Revista Electrónica de Estudios Hispánicos, 18, 5-26.

Giannakidou, A. (2011). Positive polarity items and negative polarity items: variation, licensing and compositionality. En C. Maienborn, K. von Heusinger y P. Portner (Eds.), Semantics: An international handbook of natural language meaning (pp. 16601712). Berlín: Mouton de Gruyter.

González Rodríguez, R. (2008).La polaridad positiva en español. (Tesis doctoral). Universidad Complutense de Madrid.

Hoeksema, J. (1983). Negative polarity and the comparative. Natural Language and Linguistic Theory, 1, 403-434.

Horn, L. (2002). Assertoric inertia and NPI licensing. Chicago Linguistic Society, 38, 55-83.

Kadmon, N., y Landman, F. (1993). Any. Linguistics and philosophy, 1, 3-44.

Krifka, M.(1999). At least some determiners aren't determiners. En K. Turner (Ed.), The semantics/pragmatics interface from different points of view (pp. 257-291). Holanda: Elsevier Science.

Ladusaw, W. A.(1979). Polarity sensitivity as inherent scope relations.(Tesis doctoral no publicada). University of Texas at Austin.

Lahiri, U.(1998). Focus and Negative Polarity in Hindi. Natural Language Semantics, 6,57-125.

Moreno Cabrera, J. C. (1984). Observaciones sobre la sintaxis de casi. Dicenda: Cuadernos de Filología Hispánica, 3, 239-245.

Morzycki, M.(2001). Almost and its kin, across categories. En R. Hastings, B. Jackson y Z. Zvolenszky (Eds.), Proceedings of SALT XI (pp. 306-325). Ithaka (NY): Cornell University.

Nouwen, R. (2000). Remarks on the polar orientation of 'almost'. En J. van de Weijer y B. Los (Eds.), Linguistics in the Netherlands (pp. 162-173). Ámsterdam: John Benjamins.

Pardo Llibrer, A. (2018). Los usos atenuantes del aproximativo casi: Relación entre contenido proposicional y unidades del discurso [Número Monográfico]. Estudios de Lingüistica: Universidad de Alicante, anexo 4, 33-56.

Penka, D.(2006). Almost there: the meaning of almost. Proceedings of Sinn und Bedeutung 10. ZAS Papers in Linguistics, 44, 272-286.

Pons, S. y Schwenter, S. (2011). Los significados próximo y polar de casi. En R. González Ruiz y C. Llamas Saíz (Eds.), Gramática y discurso. Nuevas aportaciones sobre particulas discursivas del español (pp. 159-188). Pamplona: EUNSA.

Real Academia Española (2009). Nueva gramática de la lengua española. Madrid: Espasa. Sadock, J. (1981). Almost. En P. Cole (Ed.), Radical pragmatics (pp. 257-271). Nueva York: Academic Press.

Sánchez López, C. (1999). La negación. En I. Bosque y V. Demonte (Dirs.), Gramática descriptiva de la lengua española (pp. 2561-2634). Madrid: Espasa. 
Spector, B. (2014). Global positive polarity items and obligatory exhaustivity. Semantics and Pragmatics, 7, 1-61.

Szabolcsi, A. (2004). Positive polarity - negative polarity. Natural Language and Linguistic Theory, 22, 409-452.

Van der Wouden, T. (1997). Negative contexts. Collocation, polarity and multiple negation. Londres: Routledge.

Ziegeler, D. (2000). What almost can reveal about counterfactual inferences. Journal of Pragmatics, 32, 1743-1776.

Ziegeler, D. (2010). Running the gauntlet on the approximatives debate: A response to recent challenges. Journal of Pragmatics, 42, 681-704.

Zwarts, F. (1998). Three types of polarity. En F. Hamm y E. Hinrichs (Eds.), Plurality and quantification (pp. 177-238). Dordrecht: Kluwer. 